\title{
Production and properties of yellow affinity substances by Ruminococcus flavefaciens
}

\author{
J Kopecny, J Simunek, B Hodrova \\ Institute of Animal Physiology and Genetics, Czech Academy of Sciences, Prague 10, Uhrineves \\ 104000, Czech Republic
}

Production of yellow affinity substance (YAS) has been described only for several strains of Clostridium thermocellum grown on cellulose (Ljungdahl et al, 1983, Curr Microbiol, 9, 195198). In this case, YAS facilitates binding of the cellulolytic complexes to cellulose and is formed prior to the complexes (Lamed et al, 1985, Enzyme Microb Technol, 7, 37-41). YAS may also be a bacterial signal substance for cellulose (Ljungdahl et al, 1988, Methods Enzymol, 160, 483-500).

Our screening of rumen cellulolytic bacteria for YAS production has led to a study of YAS formation by Ruminococcus flavefaciens strains. The highest production of yellow substances was from cultures of Ruminococcus flavefaciens 017. Growing on wheat cellulose, this bacterium produced two yellow compounds (YAS1 + YAS2) involved in cellulose degradation. These YASs were produced in early stages of growth and they could be easily extracted with acetone. In reduced form they both have a maximal absorption at $420 \mathrm{~nm}$. Similarly to the YAS of $C$. thermocellum, these compounds were sensitive to oxygen and $\mathrm{H}_{2} \mathrm{O}_{2}$ oxidation. They were involved in binding to cellulose as well as to bacterial cellulases. Adhesion of these compounds was tested on HPLC IE and RP columns. Both, YAS1 and YAS2 had a very high affinity to endoglucanase 1 and cellobiohydrolase 1 and 2 produced by the same bacterium and also for microcrystalline cellulose. In the presence of YAS1 and YAS2, an increase of endoglucanase and cellobiohydrolase activities was observed. This study shows that YASs may be more widespread in cellulolytic microorganisms than appreciated. 